\title{
La pratique autobiographique au Cercle littéraire des écrivains cheminots
}

Autobiographical writings in the french railwaymen litterary circle

José Claveizolle

\section{OpenEdition}

\section{Journals}

Édition électronique

URL : https://journals.openedition.org/rhcf/1621

DOI : 10.4000/rhcf.1621

\section{Éditeur}

Rails \& histoire

Édition imprimée

Date de publication : 10 février 2013

Pagination : 73-78

ISSN : 0996-9403

Référence électronique

José Claveizolle, "La pratique autobiographique au Cercle littéraire des écrivains cheminots », Revue d'histoire des chemins de fer [En ligne], 44 | 2013, mis en ligne le 13 novembre 2014, consulté le 22 avril 2022. URL : http://journals.openedition.org/rhcf/1621 ; DOI : https://doi.org/10.4000/rhcf.1621 
José CLAVEIZOLLE

\section{La pratique autobiographique au Cercle littéraire des écrivains cheminots}

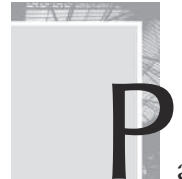

Éc. Écrivains Cheminots (CLEC), nous avons recensé une vingtaine d'ouvrages publiés à caractère autobiographique, à laquelle s'ajoutent les études diverses relatant un souvenir ou une expérience, ainsi qu' une vingtaine d'articles parus dans Le Dévorant relatant une « tranche de vie ». Nous avons pu ainsi dégager des constantes dans les écrits autobiographiques des cheminots, la famille, la hiérarchie, la guerre et le métier, que nous avons choisi d'illustrer par des extraits de cinq œuvres qui nous paraissent emblématiques.

\section{La famille}

Tous les auteurs ont conscience d'avoir négligé leur vie familiale, de n'avoir pas pris le temps de voir " pousser » leurs enfants et rendent un hommage sobre mais sincère à leurs épouses. Comme l'a dit notre regretté président d'honneur, André Escolan : " les hommes que nous sommes ne seraient pas ce qu'ils sont s'ils n'avaient pas les femmes qu'ils ont. " Le plus souvent, la raison est la passion d'un métier, l'obligation de résultat qui font peser sur eux de lourdes responsabilités et absorbent tout leur temps, ce qui n'est pas propre au monde cheminot. En revanche, il y a deux facteurs " aggravants » dus aux 
missions de la SNCF, la veille 24 heures sur 24 d'un service qui ne cesse jamais et les déplacements liés à l'implantation sur tout le territoire du réseau ferré, d'où découle cette singulière prépondérance du métier de cheminot.

\section{La hiérarchie}

Ce second point illustre une incompréhension de certaines décisions prises à distance par " ceux de la direction " auxquelles les intéressés se plient à regret. Je dois avouer que certains auteurs, que je ne citerai pas, procèdent à une critique systématique d'une hiérarchie prétendument incompétente, inhumaine, et j'en passe, ce qui rend le propos excessif. J'ai préféré illustrer ce point par le récit de Robert Rousse dans Lumière sur la voie :

En 1950, l'ingénieur « installations fixes » est chargé de remettre en état le pont mobile de Caronte, un ouvrage de 113 mètres de double voie à 23 mètres au-dessus du canal qui relie Pétang de Berre à la mer.

J'avais demandé à commander la manœuvre d'ouverture du pont depuis la gare de Martigues, située en hauteur à 200 mètres. Le câblage était prévu à cet effet mais Robert Lévi refusa :

- Pensez-vous, s'il y avait du brouillard!

- Mais, Monsieur, en cas de brouillard, la navigation est interdite.

Et puis du brouillard à Martigues! Mais ce fut la logique des brumes d'Îlede-France qui l'emporta.

Or, par un bel après-midi d'août, sous un soleil brûlant inondant la garrigue, on demanda l'ouverture du pont à la gare de Martigues pour un pétrolier. Le Marius de service répondit d'envoyer le navire et partit à vélo à la manouvre... Sous le tablier, l'eau saumâtre étincelait, aveuglante, les pneus collaient au platelage de tôles surchauffées et il voyait déjà le pétrolier avancer dans le chenal.

Malheur, pourquoi avait-il regardé ? Son pédalier vient heurter une tête de traverse ; il tombe lourdement [...] mais ce n'est pas la douleur qui fait pâlir Marius. Il tâte sa vareuse et dit :

Merde, la clé !

Dans sa chute, la clé [d'enclenchement permettant l'ouverture du pont] vient de tomber à la mer.

Que faire ? Retourner à la gare prendre la clé de secours ? Il n'a plus le temps, le pétrolier grandit et approche. Restent les signaux du pont : le pilote va bien voir que les boules noires ne sont pas descendues et que les blanches ne sont pas montées! Marius grimpe à la cabine et tend désespérément les bras.

Cependant à la passerelle du bateau, on commence à s'étonner de voir que le tablier barre la route : il pourrait se grouiller ce pontonnier endormi ! [...] Le pilote comprend soudain qu'il se passe quelque chose de grave : bon Dieu, Marche arrière toute! [...] C'est trop tard, le navire court sur son erre. 
Il va nous démâter ! crie le pilote qui gouverne droit sur la pile. La proue s'écrase sur la chaîne d'angle en maçonnerie avec un bruit ridicule de gros carton déchiré, accompagné d'un éboulis de pierres dans l'eau et sur le pont du bateau.

Pauvre Marius! S'il avait su que j'avais voulu manœuvrer son pont depuis le fauteuil de mon bureau ${ }^{1}$.

\section{La Guerre}

Les histoires relatives à la Seconde Guerre mondiale ont forcément marqué les esprits de ceux qui les ont vécues de l'intérieur avec ce statut militaire si particulier aux cheminots "d'affectation à une unité collective de défense "; autrement dit, l'obligation de rester en poste pour faire circuler les trains. Ainsi Rails et pavés, Paris août 1944 de Pierre Patin est une sorte de livre de bord, jour après jour, du 13 août au 2 septembre 1944 à Paris, entre Montparnasse (où il travaille en qualité de chef d'arrondissement Traction) et Montmartre (où il réside) : le tout à vélo et seulement armé d'un marteau d'emballeur.

Le 14 août, le chef du service MT (Matériel et Traction) convoque ses cadres pour leur apprendre que cinquante-sept cheminots sont retenus en otage et qu'ils seront fusillés si le trafic ne reprend pas. Pierre Patin et trois collègues sont volontaires pour assurer ce trafic. Seul aux commandes d'une rame banlieue, il assure d'incessants allers retours au départ de Saint-Lazare.

Le lendemain, nouvel appel :

Allo, Patin?

Je reconnais la voix du Directeur de Région Ouest, Legoux dit Charles VI à cause de son tempérament quelque peu fantasque.

- Mes respects, Monsieur le Directeur.

- Venez me voir tout de suite. Avez-vous un moyen de locomotion parce qu'il n'y a plus de métro!

- J'ai mon vélo!

- Arrivez ventre à terre.

Je saute donc sur ma bicyclette et je traverse Paris "ventre à terre ».

Charles VI, qui ne passe pas pour résister beaucoup aux exigences de la H.V.D. (Haupt Verkehrs Direktion), commence par me faire, à moi pauvre ingénieur débutant, un long discours patriotique puis m’annonce :

- Vous avez fait preuve de caractère! Voilà pourquoi je vous charge de la liaison entre la direction et les grévistes, autrement dit : la Résistance. Les Allemands vont sûrement saboter les machines en s'en allant. Il faut réoccuper les dépôts. Ils sont en force aux Batignolles et il n'y a plus un seul

1- Robert Rousse, Lumière sur la voie, Toulon, Les Presses du Midi, 1995, p. 160. 
Français. Cet après-midi à cinq heures, on va faire la paye des roulants du petit dépôt ; il faut que vous alliez les convaincre d'attaquer le dépôt le plus tôt possible ; tenez-moi au courant.

Il me donne son numéro personnel. Et voilà : c'est tout simple 2 .

C'est ainsi que Pierre Patin va reprendre le dépôt des Batignolles à la tête d'un groupe de cheminots résistants. Quelques jours plus tard, une fois passée la liesse de la libération de Paris, il procède à une tournée d'inspection du réseau Ouest à bord d'un autorail Renault. Au retour, l'heure est à la réflexion :

De retour vers Paris, la conduite laisse mes pensées vagabonder. Je me remémore le mois que je viens de vivre, les événements auxquels j’ai été mêlé, les êtres qui sont entrés dans ma vie [...] et dont la plupart en sont ressortis au bout de quelques minutes, de quelques heures ou de quelques jours. Je n'ai pas été très brave. Je me suis battu, je n'ai pas tiré un seul coup de feu. Par contre, j'ai bien failli en recevoir et, tout compte fait, j’ai eu beaucoup de chance ; beaucoup de chance aussi de pouvoir dire que je n'ai pas à avoir honte de moi ; j'ai fait ce que j'ai $\mathrm{pu}^{3}$.

De ce livre, Robert Rousse, qui était lui aussi à Paris à cette période, a écrit :

Sans doute ne faut-il pas trop assimiler les angoisses et les bravoures de ces journées parisiennes d'août 1944 à celles qu'ont pu connaître les vrais acteurs, dans les combats de chars et d'avions, ou sous les bombardements et les mitraillages directs; et Pierre Patin a raison d'écrire : "la guerre est passée sur Paris... enfin presque!"

\section{Le métier}

"Les grandes réalités du chemin de fer sont indissociables des petites chroniques des cheminots. " Ainsi Gabriel Jacquot, le conducteur du TGV du record de vitesse de 1981 (380 km/h), préface l'ouvrage autobiographique de Pierre Reure, Une vie : de la vapeur au TGV.

Entré comme apprenti, il sera l'un des derniers mécaniciens vapeur, puis suivra l'évolution du parc des machines en qualité de conducteur, avant de grimper dans la hiérarchie comme conducteur d'essai sur le TGV et l'Eurostar. Il sera marqué par la grande grève de 1986 et l'accident de la gare souterraine de Paris Lyon en juin 1988. Voici trois extraits qui illustrent ces moments forts :

En ce premier octobre 1951, c'est avec un indicible plaisir que j'entrais dans le temple de la SNCF. J'avais pour aiguillon de faire au moins aussi bien que mon frère [...] Mais, on avait joué un mauvais tour à l'apprentissage. Je m'aperçus très vite que la paupérisation de l'enseignement avait tout changé. Le célèbre et redouté directeur national de l'apprentissage,

2- Pierre Patin, Rails et pavés : Paris, août 1944, Paris, La pensée universelle, 1994, p. 44.

3- Ibid., p. 125. 
M. Grobon, [...] lança ces paroles inquiétantes que je compris plus tard : "Vous, apprentis, souvenez-vous que vous n'avez droit à rien. " Personne ne daigna nous donner d'explication et, en particulier, ce vieux bonhomme qui semblait nous mépriser [...]. On aurait pu nous dire que toutes les entreprises d'une certaine importance devaient ou former des apprentis, ou être taxées ${ }^{4}[\ldots]$.

Avec Gilbert Coquet, dit Jules, [...] spécialiste des roulements du Morvan, on redressait bien des erreurs. Le samedi, un train arrivait de Paris, avec correspondance pour Auxerre, Avallon et Clamecy. Un autorail attendait quai 5. On guettait la sortie du souterrain et, si on détectait un afflux de voyageurs, on sortait vite un renfort spécial qu’on avait préparé à toutes fins utiles. On avait une idée des affluences d'une année sur l'autre, selon les jours de fête par rapport aux week-ends, et il fallait aussi tenir compte de la météo mais il y avait toujours des impondérables. Ah, ces Parisiens imprévisibles ${ }^{5}$ !

L'accident de la gare de Lyon a provoqué la mort de 56 personnes et en a blessé 55 autres.

Il était 19 h 30 quand je me rendis à la gare souterraine de banlieue. Dès mon passage par la salle des Fresques, je fus immédiatement saisi par une ambiance peu coutumière : des files d'attente aux cabines téléphoniques et les voyageurs paraissaient stressés. Tiens, une grève sauvage ? [...] Je constatai qu'il n'y avait qu'un seul courant de voyageurs sortant de la gare souterraine par les escaliers. Je croisai Max, le visage défait : "Oh ! Si tu voyais la gare souterraine, il y a eu un accident mais j'ai vu mon fils : il est sain et sauf. " Je fonçai dans cette gare, ne réalisant pas comment un grave accident pouvait survenir dans une gare en cul-de-sac. Pas une âme! Au fond, sur la voie 4, un train de banlieue vide dont l'intérieur était éteint. J'escaladai directement sur les quais hauts et arrivai au pied d'un horrible spectacle : la motrice du train entrant avait défoncé la remorque pilote du train attendant à quai. La motrice, plus lourde, avait littéralement fendu et éventré la remorque. Le tout était encastré. Je ne distinguais que quelques corps déformés, sans vie. Pas un murmure, pas un cri, pas une plainte. Tout à coup, sorti de je ne sais où, apparut, hagard, le chef de train : " j'ai dit au conducteur : viens vite! Mais il est resté jusqu'au bout pour essayer de prévenir les voyageurs et il s'est fait tuer ! » Pas de sauveteurs, pas d'autres cheminots : on eut dit que tous les vivants avaient fui ce lieu maudit ${ }^{6}$.

Enfin, on ne peut pas parler du métier de cheminot sans évoquer l'âge de la vapeur. C'est ainsi que Pierre Patin se remémore l'incident qu'il a vécu à bord d'une $140 \mathrm{G}$ :

À cent kilomètres à l'heure, dans la descente de Saint-Mards de Fresnes, filent tout à coup au-dessus de mon épaule les deux feux rouges d'un carré fermé, non précédé de l'avertissement orange réglementaire. Le chef-mec

\footnotetext{
4- Pierre Reure, Une vie, de la vapeur au TGV : souvenirs, Paris, Éd. La Vie du Rail, 2006, p. 30.

5- Ibid., p. 107.

6- Ibid., p. 191.
} 
hurle : "Tiens bon ! »C'est le cri de détresse. Pas d'histoires : les sept manœuvres que m’a enseignées Rullier :

Le frein dans le coin.

Fermer le régulateur.

Renverser la marche.

Rouvrir le régulateur.

Ouvrir à fond les sablières.

Injecter l'eau dans les cylindres.

Allumer une cigarette en tenant bon.

C'est ce que les journalistes appellent " renverser la vapeur " et les cheminots " battre contre vapeur ". Le train s'arrête sur une distance que, le lendemain, nous mesurerons trois fois plus faible que la normale : la secousse a été sérieuse ${ }^{7}$.

Voilà un bref survol de ces témoignages, un pâle reflet de ce que nos collègues ont écrit et qui démontre que, humblement mais avec une grande conscience professionnelle, ils ont vécu leur vie de cheminot, ont fait vivre les services de la SNCF à l'image de l'immense majorité d'entre nous qui n'avons pas fait l'effort d'écrire. Le jugement personnel qu'ils portent sur l'entreprise, une fois libérés de l'obligation de réserve, pourrait bien inspirer les dirigeants actuels, telle cette remarque sur un sujet on ne peut plus d'actualité - la concurrence - formulée en 1995 par Robert Rousse, dans Lumière sur la voie :

Maintenant que les privatisations sont à l'ordre du jour, il n'est plus qu'un modèle : l'économie de marché. Qu'importe si la rentabilité est antinomique du service public, le lobby automobile commande à Bruxelles pour imposer la déréglementation où les flottes privées viendront mêler leurs tampons pour leur plus grand profit. Peut-être que ces nouveaux exploitants adopteront aussi leurs règles sociales d'amplitude des camionneurs et maquilleront leur «Flaman » pour s'aligner sur la concurrence.

\section{Références bibliographiques}

Avart Georges, De la pelle aux ficelles : une histoire du chemin de fer français, Paris, Thélès, 2003.

Brigot Daniel, Chef d'établissement SNCF 1953-1993 : mémoires d'un cheminot de l'exploitation : de l'élève au cadre supérieur, Paris, L'Harmattan, 2003. Patin Pierre, Rails et pavés : Paris, aô̂t 1944, Paris, La Pensée universelle, 1994.

Reure Pierre, Une vie, de la vapeur au TGV : souvenirs, Paris, Éd. La Vie du Rail, 2006.

Rousse Robert, Lumière sur la voie, Toulon, Les Presses du Midi, 1995.

7- Pierre Patin, Rails et pavés..., op. cit., p. 40. 